\title{
In rebus gravissimis tractandis nusquam a risu iocoque discedas: Luciano, maestro de espíritu literario (y de griego) en el Renacimiento. El caso del Momus de Leon Battista Alberti
}

\begin{abstract}
In rebus gravissimis tractandis nusquam a risu iocoque discedas: Lucian, the Teacher of Literary Spirit (and Greek Language) in the Renaissance: the Case of Leon Battista Alberti's Momus
\end{abstract}

\author{
Vicente Flores Militello ${ }^{1}$ \\ LMU Múnich - UNAM México
}

Recibido: 15-02-19

Aprobado: 03-06-19

\section{Resumen}

El presente artículo expone brevemente las circunstancias del "redescubrimiento" de la obra de Luciano en la Italia (pre)renacentista y el movimiento renovador en las letras que esto trajo consigo en el caso específico del Momus de Leon Battista Alberti. Para tal objetivo, me concentraré en el rol de Manuel Crisoloras; en los aspectos de género literario que Luciano plantea, sobre

\footnotetext{
${ }^{1}$ (vicente.flores@campus.lmu.de) Tras la licenciatura en Letras Clásicas por la UNAM México, Vicente Flores Militello prosiguió su carrera académica en la LMU Múnich, donde obtuvo el Magister Artium y, recientemente, el doctorado en Filología Latina (2018). Se ha especializado en el ámbito de la sátira romana, con particular interés en la representación literaria de fenómenos sociales en la poesía. Ha publicado un artículo sobre la cuarta sátira de Juvenal (Gymnasium 123, 2016) y está por salir su monografía sobre la representación de la relación patronus-cliens en Horacio, Marcial y Juvenal (Tubinga 2019). Ha publicado también traducciones al castellano de obras de la tradición clásica como la llamada Visio Pauli (México 2015) y el Momus de Alberti (México 2017); esta última, galardonada con el Premio para Traducciones y Difusión de la Cultura Italiana 2018 por parte del Ministerio de Bienes y Actividades Culturales (MiBAC) de Italia. Actualmente está por comenzar como investigador en el Instituto de Investigaciones Filológicas de la UNAM con un proyecto sobre la poesía política de Claudiano.
}

Araucaria. Revista Iberoamericana de Filosofia, Política, Humanidades y Relaciones Internacionales, año 21, $\mathrm{n}^{\circ} 41$. Primer semestre de 2019. Pp. 311-328. ISSN 1575-6823 e-ISSN 2340-2199 doi: 10.12795/araucaria.2019.i41.15 
todo, en el Prometheus es in verbis (la mezcla Diálogo-Comedia), y finalmente, en cómo estos son retomados en el Momus, donde Alberti se presenta como innovador de las letras latinas, pero al mismo tiempo como indirecto continuador del quehacer luciánico.

Palabras-clave: Humanismo, estudio del griego, recepción, diálogo cómico.

\begin{abstract}
This article briefly presents the circumstances of Lucian's "rediscovered" œuvre in the Italian (Pre-)Renaissance and the literary impetus which this caused in the specific case of Leon Battista Alberti's Momus. For this aim, I will focus on the role of Manuel Chrysoloras, on the aspects of literary genre which Lucian expresses above all in the Prometheus es in verbis (the mixing of Dialogue and Comedy), and, finally, on how this aspects were taken up again in the Momus, where Alberti presents himself as an innovator of Latin literature and, at the same time, as an indirect continuator of Lucian's work.
\end{abstract}

Key-words: Humanism, Greek Learning, Reception, Comic Dialogue.

\title{
EI "redescubrimiento" de Luciano en la Italia del Quattrocento
}

Me atrevería a afirmar que cualquier estudiante de griego antiguo hoy en día, sea a nivel medio o superior, ha trabajado, leyendo o traduciendo, con pasajes de Luciano de Samósata. Es, sin duda, un autor cuyos textos se adaptan muy bien a un uso didáctico ya que unen tres características muy ventajosas: una prosa correcta y aticista, un buen sentido del humor y un contenido ameno que, en su brevedad, hace referencia a varios mitos, personajes y discusiones histórico-filosóficas ampliamente conocidas, pero que también llega a reflexiones serias bastante profundas. ${ }^{2}$

Al mismo tiempo, y esto a veces quizás pase desapercibido, Luciano crea un diálogo no solo 'intracultural', en el sentido de dentro de la tradición literaria-cultural griega misma, parodiando a poetas, históricos y filósofos griegos, sino también 'intercultural' con el mundo romano en el que, a fin de cuentas, se desarrolló. ${ }^{3}$ Y si ya en la Tardía Antigüedad, hablantes nativos de griego parecen haber estudiado algo de latín con textos que podríamos decir

\footnotetext{
2 Sobre estos tres elementos v. también Ferrari 2013: 29.

3 Sobre si Luciano leía o no latín y si sus obras hacen alusión a, por ejemplo, Juvenal, cf. recientemente Manzella 2016 (en Stramaglia et al. 2016: 181-212); cf. también Rochette 1997 y Hafner 2017b: 18 n. 20. Aunque escéptico en este respecto, Swain (en Ligota/Panizza 2007: 17-44, esp. 39), sí destaca a Luciano como "a product of a multi-cultural background" (44), refiriéndose a su origen y desarrollo en los mundos sirio, griego y romano.
} 
que comparten esta característica de humor y crítica amarga, como las sátiras de Juvenal, ${ }^{4}$ debemos a Luciano buena parte del restablecimiento del diálogo profundo con los textos griegos, y la cultura que ellos implican, en el Occidente latino moderno. ${ }^{5}$

En el "redescubrimiento" europeo de la lengua y literatura griega "en original", juega precisamente Luciano un papel basilar. Su carácter didácticosatírico fue aquello que lo convirtió en el medio predilecto de enseñamiento del griego en los albores del Renacimiento: por un lado, como maestro y ejemplo de griego, gracias a su prosa aticista coloquial y elegante, así como a su contenido rico en referencias a diferentes aspectos de la cultura griega, como he dicho; por otro lado (y sobre todo, en el ámbito del género, o lo que me atrevería a llamar 'espíritu' literario), gracias a su manera de expresión y a los elementos estilísticos que caracterizan sus escritos, pues los textos de Luciano oscilan entre la seriedad de los argumentos filosóficos y lo jocoso de la burla satírica de una manera casi teatral. ${ }^{6}$

Fue precisamente con Luciano, podemos entonces afirmar, que se retomó de manera profunda el estudio formal del griego por primera vez en siglos en el occidente europeo: ${ }^{7}$ En marzo de 1396, el Canciller de Florencia, Coluccio Salutati (1331-1406; en el cargo desde 1375) envió una efusiva carta en latín a Manuel Crisoloras o Chrysolorâs ${ }^{8}$ (M. X $\rho v \sigma o \lambda \omega \rho \tilde{\alpha} \varsigma$, ca. 1350-1415), importante diplomático y erudita bizantino en Constantinopla que ya en 1390 había participado en cuestiones diplomáticas en Venecia, para invitarlo a mudarse cuanto antes a Florencia para tomar la cátedra de griego en el Studium Generale, donde lo esperarían numerosos y entusiastas discípulos. ${ }^{9}$ Crisoloras aceptó y casi un año más tarde, el 2 de febrero de 1397, llegó a Florencia. Con

${ }^{4}$ Sobre el fragmentum Antinoense, un papiro egipcio del siglo vi que reporta algunos versos de la sátira 14 de Juvenal con anotaciones en griego de carácter didáctico elementar al margen, véase recientemente Nocchi Macedo (en Stramaglia et al. 2016: 213-229).

5 Sobre los humanistas que veían en Luciano un reflejo de sí mismos, pues Luciano, como ellos, anhelaba y evocaba un pasado clásico con el que no solo se identificaba, sino en el cual se presenta y con el cual interactúa literariamente, cf. Mattioli 1980: 100 y Marsh 1998: 8 s.

6 Sobre la mezcla entre 'diálogo' y 'comedia' como característica del estilo lucianeo véase infra. Para bibliografía sobre la influencia lucianea en la literatura occidental durante y después del Renacimiento v. infra n. 13

7 Si bien ya antes había habido intentos de aprendizaje de griego, sobre todo gracias a las comunidades helenófonas del sur de la península itálica (se piense en la experiencia de Boccaccio y Petrarca con Barlaam y Leoncio Pilato a mediados del Trecento), no se había llegado muy lejos: cf. Reeve 1996: 32 s.

${ }^{8}$ En este artículo uso la forma hispanizada de su nombre. Bibliografía sobre su vida en Wulfram 2012: 89, n. 1. El texto de la carta lo reportan Reeve 1992: 133 y Wulfram 2012: 90.

9 Wulfram 2012: 90 s. subraya el carácter de prestigio político de tal decisión: La presencia de Crisoloras en Italia se entiende en el contexto de la búsqueda de alianzas políticas entre Italia y el Imperio Bizantino, pues este último se veía cada vez más amenazado por los Otomanos, quienes, como se sabe, terminaron por conquistar la Polis en 1453; de igual modo, el interés de unificar las dos Iglesias motivó a personajes de ambos lados y fomentó un intercambio cultural de grandes consecuencias. 
él llegaron, no solo nuevos aires al mundo político y cultural de la época, sino también diversos manuscritos griegos: el primero de ellos fue precisamente de Luciano. ${ }^{10}$

Crisoloras no sólo introdujo físicamente dichos manuscritos sino que trabajó con ellos para formar una nueva generación de humanistas que en menor o mayor forma asimilaron y propagaron la riqueza de la literatura griega "en original" (como lo demuestra la exponencial presencia de manuscritos griegos copiados y/o anotados después -y bajo auspicio- del maestro Crisoloras, quien incluso trabajó en una gramática didáctica del griego ${ }^{11}$ ). Así fue posible, al mismo tiempo, comprender de una manera más completa y enriquecer notablemente la literatura latina anterior y su tradición. ${ }^{12}$

Poco tiempo después de la llegada de Crisoloras a Italia, otros eruditos como el cardenal bizantino establecido en Italia, Isidoro de Kiev (1380/901463), o el humanista de origen siciliano Giovanni Aurispa (1376-1459), jugaron también un papel decisivo en la enseñanza y, sobre todo, difusión de la lengua y literatura helénica en Italia. Gracias a los numerosos manuscritos griegos que importaron de Constantinopla, entre los cuales destacan, sobre todo, la obra de Luciano, se formaron nuevas generaciones de humanistas que disponían de las herramientas intelectuales y materiales para hacer propio el pasado grecolatino, y florecieron, por sí decir, exponencialmente, primero en Italia, más tarde en el resto del occidente Europeo. ${ }^{13}$

Varios fueron los discípulos de Crisoloras: Guarino de Verona (1374-1460) y Leonardo Bruni (1369-1444), cuentan entre los más conocidos. Varios fueron también quienes, si bien quizá no participaron directamente en sus clases, sí reconocieron su enorme valor y se vieron fuertemente influenciados por sus enseñanzas: Niccolò Niccoli (1365-1437) y Poggio Bracciolini (1380-1459), son dos ejemplos importantes. ${ }^{14}$ Varios fueron también los discípulos de Aurispa e Isidoro. ${ }^{15} \mathrm{~A}$ su vez, estos humanistas transmitieron a nuevas generaciones sus conocimientos, y para finales del siglo XV Marsilio Ficino (1433-1499)

10 Berti 1985: 1987

${ }^{11}$ Los Erotemata, aunque la obra fue publicada solo en 1471. Sobre las traducciones latinas de Luciano en los siglos XV y XVI véase De Faveri 2002, cf. también Berti 2006.

12 A la mano de Crisoloras atribuye Reeve 1996: 33, por ejemplo, la corrección de los pasajes griegos en gran parte de los manuscritos de Cicerón.

13 Marsh 1998: 14 s. (en p. 33 subraya sobre todo el papel de Aurispa y los manuscritos completos de Luciano que trajera a Italia en 1423. Cf. también Mattioli 1980: 53-59). Entre las obras que tratan la tradición lucianea a partir del Renacimiento europeo destacan: Vives Coll 1959; Robinson 1979; Mattioli id.; Zappala 1990; Marsh 1998; Baumbach 2002; Furlan et al. 2007 y sobre todo los artículos de Panizza, Pantin, Bury, Deiz y Baumbach en Ligota/Panizza 2007.

${ }^{14}$ Los mayores descubrimientos filológicos del último tuvieron lugar, por cierto, durante (y a raíz de) el Concilio de Constanza, y coincidieron así con la fortuita muerte de Crisoloras en ese mismo Concilio en 1415 (sobre Poggio y Luciano cf. Marsh 1998: 32); sobre la epígrafe de la tumba de Crisoloras en Constanza y el epicedio que Poggio le dedicara v. Wulfram 2012: 94-99.

${ }_{15}$ Marsh 1998: 14 s. destaca sobre todo la influencia de Isidoro y Crisoloras en Guarino de Verona, quien tradujo a principios del siglo XV algunas obras de Luciano, así como de Aurispa. 
ya había publicado, por ejemplo, traducciones latinas de varios autores griegos y sus importantísimos tratados teológico-filosóficos neoplatónicos, y a inicios del siglo XVI, Erasmo de Róterdam (1466/69-1536) sacaba a la luz sus monumentales Adagia o el célebre Moriae encomium. ${ }^{16}$ Pero sin Luciano, cuyas obras fueron leídas en original, traducidas al latín, editadas en griego, y, sobre todo, imitadas por todos los humanistas hasta ahora mencionados, nada de esto hubiera ni siquiera imaginable. ${ }^{17}$

\section{Alberti, Luciano y el Momus}

Un caso particular sobresale entre la generación de Crisoloras y la de Erasmo: el del humanista florentino-genovés, Leon Battista Alberti (14041472) ${ }^{18}$ Además de sobresalir por sus contribuciones en el campo de la arquitectura y teoría del arte, Alberti destaca por su destreza literaria. ${ }^{19}$ En sus obras demuestra un gran interés y una particular predilección por Luciano. ${ }^{20}$ Uno de sus escritos en especial se puede considerar, incluso, como ha sido demostrado en diferentes investigaciones en las últimas décadas, como un punto de partida del género de la novela - y en particular de la novela picaresca. ${ }^{21} \mathrm{Me}$ refiero a la sátira político-filosófica Momus. $^{22}$

${ }^{16}$ Entre las publicaciones de Erasmo destacan, por cierto, varias traducciones de Luciano al latín. Los títulos latinos que él dio a la mayoría de las obras siguen siendo canónicos: Cf. Hafner 2017a: 124 n. 284.

${ }^{17}$ Sobre el rechazo que Luciano vivió gradualmente a partir del s. XVI por parte de Lutero y más tarde de la Curia Romana (en 1559 sus obras y traducciones al latín fueron incluso puestas en el Index: Zappala 1990: 163; Ferrari 2013: 29) en detrimento de figuras como Erasmo, véase el artículo de Panizza en Ligota/Panizza 2007: 71-117 (esp. 71-78). Sobre la consideración negativa que Ficino tiene contra Luciano como propagador de información falsa y denigrador de Sócrates, v. ibíd. p. 73 (con n. 6).

18 Una breve biografía ofrecen Brown/Knight 2003: XV-XVII. Para un amplio panorama de los estudios académicos sobre la biografía de Alberti v. Marsh 2002. Sobre la suerte de los escritos latinos de Alberti que pasaron por auténticos de Luciano durante buena parte del Renacimiento, v. Vian Herrero 2015: esp. 173-175.

19 Sobre Alberti arquitecto cf. Grafton 2000.

20 Alberti habría sido discípulo de griego de Aurispa, también habría sido amigo cercano de Lapo da Castiglionchio, el joven (ca. 1405-1435) y Rinuccio da Castiglione (ca. 1395-ca. 1456). Estos últimos tradujeron varias obras de Luciano al latín, y ejercieron gran influencia en Alberti: Marsh 1998, 35-37. Rinuccio es también conocido por su traducción latina de las fábulas de Esopo (Marsh ídem.; cf. también Marsh 2004). Sobre Alberti como experto de Luciano cf. también Robinson 1979: 86-94.

21 Sobre Alberti como precursor de la novela v. Zappala 1990: 67-71 (esp. 71); como precursor del género picaresco v. Marsh 1998: 127 ss.; 161; Cros 1967: 239; Zappala id.: 203 (que se remonta también al De parasito de Luciano). Sobre el particular éxito que tuvo esta obra en España cf. también Vives Coll 1959; Zappala id.: 167-190. Para el Momus como obra precursora de la “'black' comedy of hypocresy" Marsh 1998: 129.

22 Para las ediciones y traducciones modernas de la obra véase el apartado en la bibliografía. Entre las ediciones y estudios más recientes del Momus albertiano destacan Brown/Knight 2003, que introdujo la numeración de los párrafos, y Furlan et al. 2007, cuyo texto citaré en este artículo. 
Por su influencia lucianea, se llega a considerar el Momus como una obra precursora también del género fantástico. ${ }^{23} \mathrm{Y}$ en efecto, presenta las aventuras del personaje homónimo de la mitología griega en un pasado mítico y pseudohistórico, con tanto de transformaciones, apariciones y sorpresas (y referencias literarias), que son del todo fantásticas. Un tono de crítica irónica ${ }^{24}$ hacia la Corte Olímpica, su príncipe supremo, Júpiter, y el quehacer humano en la Tierra, en particular el quehacer de los filósofos, es lo que, además, emana del texto: Momo es una figura nefasta de la mitología griega; con sus acciones e intrigas termina por poner al género humano al borde de la destrucción total, pues convence a Júpiter de la necesidad de eliminar a los mortales y su entorno para volver a crear un nuevo y mejor mundo.

Por un lado, la obra mira a criticar la ambición y la hipocresía de todos los personajes, divinos y mortales; por el otro, el autor insiste repetidamente en recalcar la búsqueda de una novedad creativa en el tema y la manera de presentar los argumentos oscilando entre efectos teatrales cómicos y un discurso ético más bien serio.

Sobre este último aspecto, Marsh (1998) nota la influencia crucial de los escritos de Luciano y habla sobre "new forms of imaginative fictionshybrids of satirical narrative and comedic dialogue that gave humanists a greater freedom of invention in Latin and vernacular prose" ${ }^{25} \mathrm{Y}$ en efecto, si se consideran diversos Diálogos, y otros escritos como Charon o Contemplantes, Icaromenippus, Iuppiter Tragoedus, Nigrinus, Verae historiae y, sobre todo, el breve escrito Prometheus es in verbis, resulta evidente el ejemplo lucianeo como modelo e inspiración del contenido del Momus, pero sobre todo, como modelo de la forma: Alberti se propone mezclar efectos casi burlescos con argumentos de tono serio moral para suscitar la risa de su lector y al mismo tiempo invitarlo a la reflexión. ${ }^{26}$ Esto se puede observar ya en el proemio del Momus así como en el primer párrafo que abre cada uno de los cuatro libros que lo componen. Allí Alberti recuerda a su lector casi con insistencia el interés que tiene en unir lo divertido a lo serio y la reflexión. Por ejemplo se puede citar un breve pasaje del proemio (Momus prooem. 5):

Nos contra elaboravimus ut qui nos legant rideant, aliaque ex parte sentiant se versari in rerum pervestigatione atque explicatione utili et minime aspernanda.

\footnotetext{
${ }^{23}$ Cf. Ferrari 2013: 28 s.

${ }^{24}$ Para el concepto de ironía en Alberti y Luciano, v. Marsh 1998: 125 ss. Para la ironía socráticasatírica de Luciano v. Deriu 2017: 87-97.

25 Marsh 1998: 11 s.: La obra de Luciano como medio de innovación en la literatura del Quattrocento, muy marcada aún por el antecedente trecentesco de Petrarca. Cf. también Marsh 1980: esp. 78 ss.

${ }^{26}$ Furlan et al. 2007: XVIII s. notan tanto el carácter de filosófico político del Momus como la influencia lucianea que moldeó el espíritu literario en Occidente.
} 
Nosotros, por nuestra parte, trabajamos de modo tal que quienes nos lean, rían; pero por otra parte, vean que están en una explicación de argumentos y en una investigación útil y nada despreciable. ${ }^{27}$

Convendría exponer, entonces, brevemente el Momus albertiano y el escrito de Luciano Prometheus es in verbis para poder mostrar en consecuencia lo lucianeo e innovador en Alberti.

\section{Contenido y forma del Momus de Alberti en diálogo con el Prometheus es de Luciano}

El Momus se divide en un proemio dedicado a tú anónimo ${ }^{28}$ y cuatro libros. Alberti trabajó en su composición entre 1443 y 1450; aunque la obra circuló en forma manuscrita hasta 1520 cuando las dos primeras ediciones vieron la luz en Roma (editores: Étienne Guillery y Giacomo Mazzocchi, respectivamente) ${ }^{29}$.

En Momus se narra, entonces, la creación del mundo a manos de los dioses y las diversas luchas entre ellos por el quehacer y destino de la humanidad, de la cual sacan gran provecho. En el centro de la crítica está la disimulación, y en cierto grado, hipocresía, que caracteriza a todos los personajes: Momo es un dios feo y criticón y causa gran antipatía entre los demás dioses. Tras verse en los primeros dos libros como personaje nefasto y aborrecido y, por ende, desterrado a la Tierra, Momo, cambia táctica en el libro tercero (por un lado, en la Tierra había experimentado en carne propia que su actitud rencillosa tampoco le traería nada bueno entre los mortales; por el otro, los dioses veían que Momo los hacía peligrar más estando en la Tierra de lo que lo hacía antes en el Olimpo, así que Júpiter decide mandarlo llamar) y comienza a simular un cambio de índole para ganarse la gracia de Júpiter. Pero por burlarse (no tan) disimuladamente de los dioses y de los hombres, cae en sinnúmero de peripecias y rencillas: Terminará arruinado, mutilado, desterrado nuevamente del Olimpo y convertido en informe peñasco en medio del mar (incluso su nombre terminará mutilado al final en, alegóricamente, humus ('tierra'): Momus 3,75). En el cuarto y último libro se narran las peripecias que los dioses viven en la Tierra como consecuencia de las intrigas de Momo (aquí destacan

${ }^{27}$ Traducción propia, ligeramente adaptada de Flores Militello 2017.

${ }^{28}$ Probablemente el "tú" anónimo del proemio se refiriere al entonces gobernador de Ferrara y amigo personal de Alberti, Leonello d'Este, quien había sido ya destinatario de otros escritos albertianos; como murió antes de la conclusión del Momus, habría sido omitido: cf. Rinaldi 1986-87: $138 \mathrm{~s}$.

${ }^{29}$ A partir del siglo XVI aparece en algunos manuscritos (e incluso en la edición de Guillery) como título alternativo la denominación De principe, pues la obra se concentra en la función regente de Júpiter como príncipe o gobernante supremo -sin embargo, nada parece indicar que Alberti mismo haya dado este subtítulo a la obra-.

Araucaria. Revista Iberoamericana de Filosofia, Politica, Humanidades y Relaciones Internacionales, año $21, \mathrm{n}^{\circ} 41$. Primer semestre de 2019. Pp. 311-328. ISSN 1575-6823 e-ISSN 2340-2199 doi: 10.12795/araucaria.2019.i41.15 
los viajes de Caronte y su 'lucianesco' acompañante, el 'divertido' filósofo Gelasto, que recuerdan a Caronte y Hermes en el Charon o Contemplantes de Luciano); así concluye la obra con una retrospectiva más bien amarga, primero por parte de Momo sobre sus propias experiencias (Momus 4,79-89), y segundo por parte de Júpiter mismo, sobre su propia falta de visión (Momus 4,99-102).

La inspiración del material, es decir, el contenido, viene claramente de Luciano: Si bien Momo, la personificación del reproche (en griego: $\mu \tilde{\omega} \mu$ os;

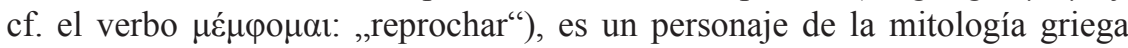
ya presente en la Teogonía de Hesíodo, ${ }^{30}$ es sobre todo en Luciano que Momo encuentra el culmen de su representación literaria con casi cuarenta apariciones: ${ }^{31}$ En obras como Iuppiter Tragoedus o Deorum concilium, Momo es el personaje interlocutor de Zeus sobre distintos temas de filosofía y vida divina. En Nigrinus (32), Verae historiae (2,3), Icaromenippus (18), Dearum iudicium (2), y finalmente, en Hermotimus (20) y en Bacchus (8) - Momo es una figura de referencia central, y los detalles que Luciano le atribuye (por ejemplo: el considerar ridículo que los toros tuvieran los cuernos sobre la cabeza y no bajo los ojos: Nigr. 32; hist. 2,3), son retomados como subtexto por Alberti de modo que el efecto es bastante cómico (cf. Momus 1,$7 ;^{32} \mathrm{cf}$. también el excurso sobre la alabanza de la vida como vagabundo en Momus 2,47-58, que retoma en gran medida el escrito De parasito de Luciano). ${ }^{33}$

Por otro lado, en varios Dialogi mortuorum así como en Menippus, Icaromenippus, Cataplus, y sobre todo en Charon o Contemplantes, personajes del mundo mitológico e histórico, frecuentemente filósofos, se encuentran en las situaciones más inimaginables. Alberti las retoma de nuevo con gran cercanía y destreza: Mientras Caronte discute en Luciano con Hermes, por ejemplo, en escenarios chuscamente improbables sobre reyes y zapateros, sobre la vanidad del mundo y la igualdad de la muerte; en Alberti (Momus 4), lo hace con el filósofo Gelasto, quien lo guía por el mundo de los mortales; el filósofo cínico Menipo sube volando al Olimpo en Luciano, como Ícaro al cielo, para enterarse de los planes de Zeus de destruir a los filósofos; son precisamente estos planes a los que se alude en el Momus albertiano (Momus 2,109).

En cuanto a la forma, es decir la lengua que Alberti usa y el sentido global que le da a la obra, Momus está escrito en una prosa latina elegante

${ }^{30}$ Hesíodo (Th. 214f.) presenta a Momo como hijo de la noche (Nv́ł) y hermano, entre otras figuras, de la Miseria ('Ö̌̌ús). Calímaco (Ap. 113) lo apostrofa y lo exhorta a irse donde está la Envidia ( $\Phi \theta$ óvoऽ). Se vuelve tópico como figura de censura y reprensión, como muestra un pasaje en la República de Platón (487a). También en el mundo romano aparece en sentido similar: En el tratado De natura deorum $(3,44)$, Cicerón lo interpreta como la figura de Disputa (Querella); en un pasaje de las Epístolas a Ático $(50,20,6)$, Momo representa de forma sarcástica la censura y la tosquedad.

${ }^{31}$ Para un panorama sinóptico completo de las obras de Luciano en el Momus v. sobre todo Mattioli 1980: 91-100; Rinaldi 1986-87 y Marsh 1998: 117-129.

32 Tema también de una fábula de Esopo (102), autor igualmente "reintroducido" en griego en los albores del Quattrocento. Sobre esto v. supra n. 14.

33 Sobre la alabanza a la vida del vagabundo en Momus y Luciano véase Marsh 1998: 161. 
y correcta que, sin embargo, tiende a la complejidad debido a periodos largos y rebuscados, ${ }^{34}$ así como a un sinnúmero de alegorías. ${ }^{35}$ La extensión de la obra es mayor a cualquiera de Luciano. El texto albertiano tiene varios excursos, seguido terminan complejos cuestionamientos filosóficos de manera abrupta, porque el interlocutor se harta o la escena es interrumpida por algún acontecimiento imprevisto (lo que causa un efecto paródico bien logrado), son frecuentes las escenas chuscas (con contenido escatológico, aunque con lenguaje refinado), etc.

Recuerda en algunos aspectos también a Apuleyo, igualmente un autor importante de la Segunda Sofística (contemporáneo, es decir, de Luciano, y, podríamos decir, primer receptor suyo en el mundo latino $\left.{ }^{36}\right)$ : el lector encuentra así, por un lado, un alternar incesable de historias y escenas increíbles, caracterizadas por violencia, detalles morbosos, fantasmas, aventuras, etc., por el otro, una trama que acompaña a los respectivos personajes a través de un iter filosófico con un fondo de humorismo evidente y una elección lexical y sintáctica muy refinada. No obstante, el mayor eco en el texto de Alberti es el de los escritos de Luciano: Muy lucianeo es, por ejemplo, el espíritu de citar, parodiando, ${ }^{37}$ a diversos autores de la tradición clásica - desde discursos filosóficos en tono aristotélico y platónico en boca de los mismos filósofos, hasta citas textuales de poetas, en este caso: Persio, Marcial y Juvenal ${ }^{38}$ (autores que, cabe recordar, comparten el interés satírico con Luciano pocas generaciones antes que él). Sin embargo, es sobre todo una característica la de mayor peso: la ya mencionada insistencia de Alberti como autor en querer justificar con el lector la elección del tema y la manera de presentarlo; es decir: lo que él llama la novedad en las letras latinas de unir la broma a los argumentos serios en una prosa refinada de manera convincente.

${ }^{34}$ Marsh 1980: 78 ss. y sobre todo 1998: 130 nota la tensión en el lenguaje latino humanista en general, donde el sermo cottidianus de la comedia (Terencio, Plauto) y la Latinitas (más o menos) ciceroniana contrastan maravillosamente con los nuevos modelos lucianeos.

${ }^{35}$ Cf. Ferrari 2017: 29. Se piense, por ejemplo, en la caracterización de los hijos de Virtud hacia finales del libro 1 (sobre todo su huida de la Tierra en Momus 1,96): Trofeo, Triunfo, Posteridad y Alabanza, o en el fruto de la violación de esta última por parte de Momo/Reproche (Momus 1,74): el monstruoso Chisme (Fama).

${ }^{36}$ Se piense en el Onos y las Metamorfosis. Cf. Marsh 1998: 128 (con n. 74) quien advierte ya en el De oratione libri septem de Antonio Lulio (1558) la influencia de Apuleyo y Luciano sobre, por ejemplo, la novela picaresca y el género del "poema quod vocant dramaticum".

37 Se podría entender 'parodia' como la adaptación de aspectos lingüísticos y estilísticos en un nuevo contexto con fines cómicos: cf. Quint. 9,2,35.

38 Para las parodias a Sócrates, Platón, Aristóteles, Teofrasto, entre otros cf. Mattioli 1980: 95 y sobre todo Rinaldi 1986-86: esp. 142 ss. Sobre los satíricos romanos: En Momus sus versos son presentados como absurdas versiones del oráculo de Apolo o como confutación de la existencia de los dioses (Juvenal 7,81; 103 y Persio 3,49 s. como oráculos en Momus 3,30; una variación de Marcial 8,24,5-6, como "prueba" de la no existencia de los dioses en la escena escatológica del teatro: Momus $4,18)$.

Araucaria. Revista Iberoamericana de Filosofia, Política, Humanidades y Relaciones Internacionales, año 21, $\mathrm{n}^{\circ} 41$. Primer semestre de 2019. Pp. 311-328. ISSN 1575-6823 e-ISSN 2340-2199 doi: 10.12795/araucaria.2019.i41.15 


\section{Prometeo y Luciano en palabras: la correcta mezcla de 'Diálogo' y 'Comedia' y el 'espíritu' literario en el Momus de Alberti}

En tres breves escritos, Zeuxis, Bis accusatus y Prometheus es in verbis, Luciano tematiza los problemas de su revolucionario estilo literario; es decir, los cuestionamientos que él plantea que su forma novedosa de escribir, que mezcla diversos elementos de géneros distintos (lo que yo llamo aquí 'espíritu' literario) le han traído. En Prometheus es in verbis o como se suele llamar también, Prometeo literario, ${ }^{39}$ el autor justifica lúdicamente ${ }^{40}$ su estilo o 'espíritu' literario ante una comparación que se le hace con Prometeo, quien de barro creara a los hombres, engañando a los dioses y poniendo en peligro su proprio destino. Luciano indica que sospecha una mofa irónica: ${ }^{41}$ Sus obras serían cacharros raros sin gran valor. ${ }^{42}$

Luciano responde con ambigüedad en lo que sigue de la obra, aunque se muestra seguro y orgulloso de haber siempre presentado textos innovadores

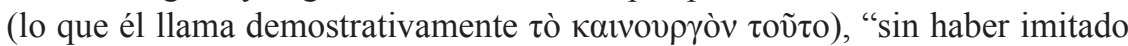

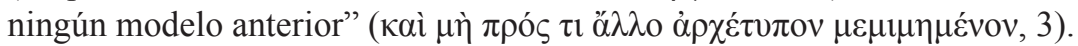

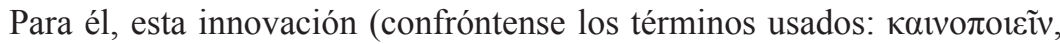

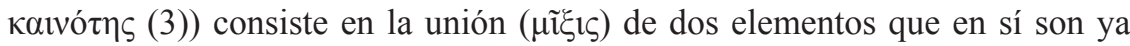
completos y de gran valor - de ahí la dificultad -, es decir: la unión "de los

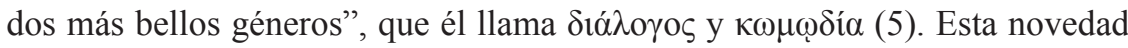
representa, sin embargo, al mismo tiempo un peligro: Por un lado, Luciano se muestra preocupado de crear monstruosos hipocentauros literarios. ${ }^{43}$ Por el

39 Mattioli 1980: 97 lo describe como: "un poco l'ars poetica del samosatense". Hopkins 2008: 109-118 ofrece un comentario de la obra; Ní Mheallaigh 2014: 1-8, un interesante análisis que explota las miras artístico-literarias de Luciano en cuanto a autor de la Época Imperial y sobre todo su actualidad "(post)moderna". Baumbach/von Möllendorf 2017 toman la obra como punto de partida para un análisis de la vida, obra y recepción de Luciano y la Segunda Sofística. Igualmente, Deriu 2017 se basa sobre todo en el rol estructural de la mixis y la poikilia en los tres escritos mencionados para un análisis de la visión crítica y la técnica de comedia aristofánica en Luciano. Sobre este tema cf. también Camerotto 2014 (esp. 109-169) (recensión: Hafner 2016). Fundamental aquí es también el volumen de Marquis/Billault 2017 (recensión: Deriu 2018).

${ }^{40}$ Sobre el juego de palabras $\pi \alpha 1 \delta$ ió- $\pi \alpha 1 \delta \varepsilon i ́ \alpha$ ('juego'-‘cultura') en este escrito cf. Brusuelas 2009, quien nota bien, además, el vínculo con el Humanismo.

${ }^{41}$ Luciano se muestra desde el inicio consciente de la ironía y sarcasmo (habla claramente de

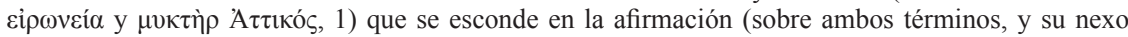
intrínseco con la figura de Sócrates, ateniense e cípov por antonomasia, v. Hopkins 2008: ad loc.).

42 'Prometeos' se llamarían en Atenas también a los alfareros, subraya Luciano mismo (Prom. es 2). Sobre el vínculo que Luciano establece entre sus obras literarias y las obras de barro en general, v. Hopkins 2009: ad loc.

43 El hipocentauro es una figura que Luciano usa aquí para ilustrar la mezcla híbrida entre de la seriedad del diálogo y la diversión de la comedia. En Zeuxis representan el resultado la destreza artística tanto del pintor (Zeuxis) como de Luciano; en el Bis accusatus Luciano se identifica con

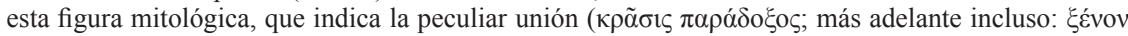

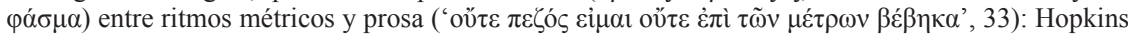
2009: 109 s. Cf. también Braun 1994: 354ss; Ní Mheallaigh 2014: 3 s. y Baumbach/von Möllendorf 2017: 171 ss.

Araucaria. Revista Iberoamericana de Filosofia, Política, Humanidades y Relaciones Internacionales, año $21, \mathrm{n}^{\circ} 41$. Primer semestre de 2019. Pp. 311-328. ISSN 1575-6823 e-ISSN 2340-2199 doi: 10.12795/araucaria.2019.i41.15 
otro, es consciente de que la novedad por sí sola no garantiza la calidad del texto, pues condiciones para que tal mezcla resulte con éxito son la armonía y la proporción. ${ }^{44}$ Poco más adelante (6), Luciano explica personificando ambos elementos, qué entiende por 'diálogo', y qué por 'comedia': Mientras que 'diálogo' se refiere a la literatura más bien seria del tipo filosófico ${ }^{45}$ (y en prosa), que se ocupa de las más elevadas cuestiones de la filosofía, como la esencia de la naturaleza y de la virtud, 'comedia' se refiere a la literatura festiva, en verso, que, acompañada de música en el teatro de Dioniso, no perdona la burla ${ }^{46}$ a los personajes serios, a quienes se inclinan a cultivar el diálogo - lo que podríamos llamar una burla satírica a la Aristófanes, por ejemplo en las Nubes. ${ }^{47}$

Diálogo y Comedia están así en diametral oposición; sin embargo, Luciano como artista, si bien intenta presentarse modesto al no mostrarse seguro del éxito de sus esfuerzos, sí subraya el valor que tiene el atrevimiento en sí de haber puesto en armonía estos dos elementos y hace evidente su gran mérito, sobre todo recalcando la originalidad de sus escritos. ${ }^{48}$

Alberti retoma este 'espíritu' literario de diálogo cómico sobre todo en el Momus, y se coloca como innovador de las letras latinas. No nombra a Luciano directamente pero para el lector atento, el eco es claro. ${ }^{49} \mathrm{Si}$ Luciano se veía como un Prometeo en palabras, podríamos decir que Alberti lo hacía, como un Luciano en latín. ${ }^{50}$

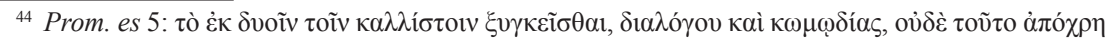

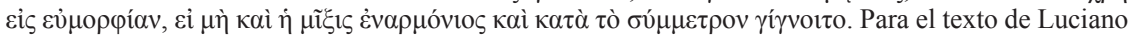
sigo la edición de Macleod 1987.

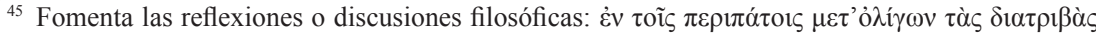

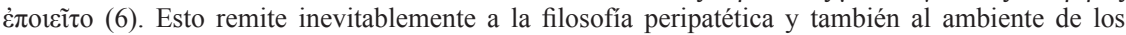
diálogos platónicos; más adelante la especifica como "el filosofar sobre las más elevadas cuestiones sobre la naturaleza y la virtud" (6: v. infra nota siguiente).

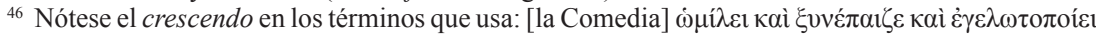

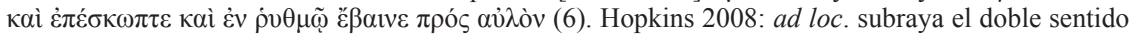
con fondo sexual en estos verbos, cosa que se vería confirmada por las descripciones que siguen en el texto.

${ }^{47}$ Cf. Deriu 2017: 12-75, aunque ella se concentra más en los elementos formales de comedia aristofánica en Luciano y subraya la función de crítica que, por ejemplo, en el Charon tiene la risa que suscita Caronte en el público (53 ss.) y cómo este último termina involucrado (60 ss.), y la compara con las Ranas. V. infra n. 57.

${ }^{48}$ Con una pointe cierra Luciano el escrito, asegurando que si efectivamente él fuera un Prometeo literario, por crear nuevos textos, al menos él, Luciano, no ha engañado a su público como en cambio Prometeo engañó a Zeus con las ofrendas de huesos recubiertas con grasa (cf. Hes. erga 49 ss.; Th. 565 ss.), es decir, no ha cometido ningún plagio literario (cf. Hopkins 2009: 109) - podrá ser un Prometeo, pero de ninguna forma un débil Epimeteo. Como nota Ní Mheallaigh 2014: 8: "this is what makes Prometheus the ideal icon for Lucian's modernity: because his mythology represents, not just the conflict between generations but, more subversively, it opens up the unsettling possibility that the mimetic rebels may, in fact, be more original than their predecessors. Prometheus, in Lucian's hands, becomes a champion of a defiant and inventive postclassical poetics."

${ }^{49}$ Cf. Mattioli 1980: 97 s. (quien remite a Martini 1942). Véase también: Marsh 1998: 117.

${ }^{50}$ Ferrari 2013: 29 tiene razón al advertir que Alberti más bien tomó impulsos estilísticos y motivos concretos (einzelne stilistische und motivische Züge) de la obra lucianea para el Momus. Dicha influencia concreta es, sin embargo, de gran peso. 
Ya en el proemio del Momus se plantea Alberti la dificultad de tratar temas novedosos, pues la gran tradición clásica se habría ocupado de casi todo. Pero aunque el tratar argumentos "nuevos e inauditos" (res novas, inauditas) sería algo extraordinario, casi igualmente extraordinario sería presentar argumentos quizás conocidos, pero de una forma novedosa (novo quodam et insperato

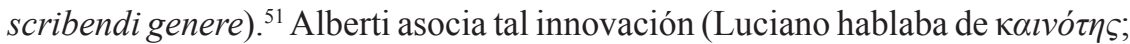
Alberti, de novitas) también a la raritas (Momus prooem. 2; 5) en el sentido de que es algo 'poco frecuente y exquisito', y se basa en el efecto cómico que la $\pi$ oıkı $\lambda$ ía de argumentos, o bien la varietas rerum (Momus 3,1), genera gracias a la interacción de bromas y eventos chuscos o inesperados con temas serios que llevan a la reflexión y contribuyen a un filosófico bene vivere (vivendi ratio et modus)..$^{52}$

Este 'espíritu' literario tiene, entonces, la característica de instruir al lector con argumentos serios y de carácter más bien ético; al mismo tiempo, de entretenerlo por medio del sentido del humor con bromas para provocar su risa. Y es precisamente en este punto donde Alberti se presenta indirectamente como seguidor de Luciano, pues - recalca -, esta técnica literaria, es decir, este 'espíritu', no ha sido casi explotado por los autores Latinos anteriores él (Momus prooem. 4):

Itaque sic deputo: nam si dabitur quispiam olim qui cum legentes ad frugem vitae melioris instruat atque instituat dictorum gravitate rerumque dignitate varia et eleganti idemque una risu illectet, iocis delectet, voluptate detineat - quod apud Latinos qui adhuc fecerint nondum satis extitere - hunc profecto inter plebeios minime censendum esse.

Así pues, considero que si llegara a haber alguien que instruyese y enseñase a sus lectores a gozar de una vida mejor con la seriedad de las expresiones y la dignidad varia y elegante del contenido, y al mismo tiempo encante con el humor, entretenga con bromas y sea placentero (cosa que entre los autores latinos que hasta ahora han escrito, no hay muchos), a este, no hay que considerarlo de ningún modo entre los autores banales.

Evidentemente, al decir que tal manera de tratar argumentos nondum satis extitere, atenúa Alberti su afirmación de innovación. Por un lado, alude a sus inmediatos predecesores, compañeros discípulos y maestros que tradujeron e imitaron en latín a Luciano, por ejemplo; por el otro, también remite a las máximas horacianas como omne tulit punctum, qui miscuit utile dulci / lectorem delectando

\footnotetext{
${ }^{51}$ Momus prooem. 4.

${ }_{52}$ Momus 3,1: Superiores, credo, libri rerum varietate et iocis delectarunt: fuit etiam quippiam in illis, ut videre licuit, quod quidem ad vivendi rationem et modum conferat. Qui sequentur libri nulla erunt ex parte aut iocorum copia aut insperatarum rerum eventu et novitate superioribus postponendi et, ni fallor, eo erunt fortasse hi anteponendi superioribus, quo maiora atque digniora recensebuntur.
} 
pariterque monendo o ridentem dicere verum, quid vetat ${ }^{53}$ Sin embargo, al poner énfasis en apud Latinos, implica más que nada a los griegos - y en este caso particular, a Luciano. ${ }^{54}$ Igual que Luciano en el Prometheus in verbis, se muestra aquí Alberti modesto en cuanto al resultado, pero sí seguro de su esfuerzo: este 'diálogo cómico' implica una dificultad enorme que se puede alcanzar solo con studium y diligentia (5). Similar a Luciano es también la defensa de Alberti hacia posibles detractores ${ }^{55}$ (Momus prooem. 5):

Cuperem in me tantum esset ingenii quantum in hac una re procul dubio difficili assequenda adhibui studii et diligentiae. Nam fortassis essem assecutus ut apertius intelligeres versari me in quodam philosophandi genere minime aspernando. Et didici quidem ipsa ex re quantum industriae debeatur ubi te studeas esse quovis pacto dissimilem ceteris dignitate et gravitate servata. Sin vero a te susceperis ita scribere ut in rebus gravissimis tractandis nusquam a risu iocoque discedas cum insueto tum et digno et liberali profecto, illic plus laboris et difficultatis invenies quam inexperti opinentur.

Desearía tener tanto ingenio, cuanto celo y diligencia apliqué en este único objetivo, que sin duda es difícil de conseguir. Pues tal vez hubiera logrado que entendieras mejor que estaba yo en una especie de genero filosófico nada despreciable. También he aprendido por el argumento mismo cuánto esfuerzo se necesita si te propones ser diferente a los demás a como dé lugar, mas conservando la dignidad y la seriedad. Pero si te propones escribir así, es decir, que al tratar argumentos de gran seriedad, nunca te alejes del humor y del juego - con un estilo insólito, pero también elegante y completamente refinado - encontrarás más trabajo y dificultad de lo que pensarían los que no tienen experiencia.

Si bien Alberti parece dar más peso que Luciano a las cualidades del diálogo, es decir, al valor ético al afirmar que se encuentra in quodam philosophandi genere, donde dignitas y gravitas juegan un papel central, ${ }^{56} \mathrm{de}$ todos modos, para ambos autores, el efecto cómico es el principal medio por el cual no solo hacer evidente su destreza literaria, sino también transmitir su mensaje, de carácter didáctico-moral-literario. Esto lo demuestra reiterando en el último párrafo del proemio y en el primer párrafo de cada libro que su obra (que él llama historia) consta de bromas (para esto usa términos como iocus,

${ }^{53}$ Respectivamente: Hor. ars 343 s. y sat. 1,1,24. En este sentido también se aludiría a la sátira menipea - o lo que queda de ella: la Apocolocíntosis de Séneca o el Satiricón de Petronio, por ejemplo.

${ }^{54}$ Mattioli 1980: 97; Marsh 1998: 117. Cf. también Marsh ibíd.: 10: "Since his works [de Luciano] are written in straight-forward and colloquial prose, he could be easily translated and assimilated by humanist students. His methods of viewing the world likewise proved more congenial and adaptable than those of Roman satire."

55 Sobre el defenderse ante personajes ficticios como recurso literario de carácter programático muy frecuente en Luciano v. Hafner 2017b: 23-36 (esp. 33 ss).

${ }^{56}$ Considérese la definición que da a su obra poco más delante como in rerum pervestigatione atque explicatione utili et minime aspernanda (pasaje citado supra en el texto de la n. 27).

Araucaria. Revista Iberoamericana de Filosofia, Politica, Humanidades y Relaciones Internacionales, año $21, \mathrm{n}^{\circ} 41$. Primer semestre de 2019. Pp. 311-328. ISSN 1575-6823 e-ISSN 2340-2199 doi: 10.12795/araucaria.2019.i41.15 
risus, sales, comitas, etc.) y que su objetivo es hacer reír a su lector ${ }^{57}$ (Momus prooem. 9):

\begin{abstract}
Sed de his hactenus. Ceterum cum tu nos per otium legeris et tibi inter legendum res ex desiderio meo, tua pro expectatione successerit, totiens congratulabimur quotiens incideris ut rideas. Et utinam tam saepe eveniat ut sales et inventorum formas admireris quam non interraro dabitur ut rideas iocos et comitatem quibus haec historia refertissima est. Ergo lege vel maxime ut ipsum te recrees, proxime ut faveas et studiis et lucubrationibus nostris volens ac lubens. Sis felix.
\end{abstract}

Pero basta de esto. De todos modos, si me lees en momentos de ocio y tienes éxito durante la lectura - como yo lo deseo y tú lo esperas - tantas veces me alegraré, cuantas te suceda reír. Y espero que esto pase, por un lado, tan seguido, que puedas admirar la sal y las formas de mis invenciones y que, por el otro, haya no pocas oportunidades de reírse de los bromas y la gracia con que está llenísima esta historia. Entonces, lee, primero para que te diviertas a ti mismo; segundo, para que te muestres favorable a mis esfuerzos y elucubraciones con ganas y alegría. ¡Séme propicio! ${ }^{58}$

\title{
Conclusión
}

Gracias al papel didáctico que Crisoloras primero, Aurispa e Isidoro de Kiev después, dieron a Luciano en el Quattrocento italiano, se formaron generaciones de Humanistas que no solo leían en griego, sino que imitaban también y hacían propios a los autores "redescubiertos"-el primero de todos: Luciano-. Esto redundó en un enriquecimiento inmenso para la literatura del occidente latino y vernáculo. El caso del Momus albertiano en particular, es un claro ejemplo de que el 'espíritu' literario quedó profundamente marcado por la influencia de Luciano. La manera que para Alberti es tan novedosa de presentar argumentos serios con elementos chuscos en una prosa amena y que hace tantas referencias a distintos niveles literarios (desde citas textuales y paráfrasis de autores antiguos, hasta parodias de personajes del mito y la historia de la filosofía griega) es del todo lucianea ('diálogo cómico'), como la

${ }^{57}$ Camerotto 2016: 285-323 se concentra en "il riso dell'eroe satirico e i suoi effetti" (285) en Luciano, que termina por involucrar al lector como partícipe de la burla crítica. Deriu 2017 analiza la función estructural de la risa. Por ejemplo, en el Charon nota cómo la risa que los personajes desencadenan en el lector instaura "un rapporto con il pubblico, che partecipa al processo di denuncia" (126). Ahora, puede notarse aquí cómo Alberti sigue los pasos de Luciano también en este aspecto.

${ }^{58}$ En este sentido se entiende mejor el cierre del proemio con el sis felix, que traduzco como pío-cómico "séme propicio", más que un llano "sé feliz", tomando en consideración el carácter propiciatorio que esta expresión tiene en la poesía latina, sobre todo como invocación a divinidades cuales Apolo o Diana (en la oración anterior pide de hecho Alberti a su destinatario mostrarse favorable: faveas). 
comparación con algunos pasajes en este trabajo, sobre todo del Prometheus es in verbis, ha demostrado: si Luciano establece un paralelo explícito entre la figura del mítico Prometeo, creador de los hombres, y su persona como artista, creador de un nuevo estilo literario que une la seriedad del diálogo filosófico en prosa y la burla satírica de la comedia ática en verso, así también se presenta implícitamente Alberti en el Momus como un Luciano latino que une la seriedad de los argumentos filosóficos con la burla satírica en una prosa latina erudita y alegre.

En el proemio al Momus (5), Alberti, y este es el título que elegí para el presente artículo, reconoce la dificultad de "nunca dejar la risa y el juego al tratar argumentos de gran seriedad" (in rebus gravissimis tractandis nusquam a risu iocoque discedas), pero ve en esto la forma de sobresalir como autor. Al hacer reír a su lector presentado una gran variedad de temas y situaciones jocosas sobre un argumento que en el fondo es serio (la crítica política y filosófica al poder y a la sociedad), logra su objetivo. El resultado del ejemplo lucianeo es un autor que ve con cierto orgullo el fruto de su esfuerzo, y esto es un texto satírico de gran valor y complejidad, lleno de alegorías, discursos éticos y elementos fantásticos; al mismo tiempo, lleno de situaciones chuscas, burlas y de gran comicidad. Al retomar este quehacer luciánico, como lo podríamos llamar, Alberti inaugura, por así decir, un nuevo 'espíritu' literario de grandes repercusiones en el mundo de las letras - y que, hoy en día, sigue muy presente. ${ }^{59}$

\footnotetext{
${ }^{59}$ Agradezco la invitación que Markus Hafner y Maria Gerolemou me han hecho para participar
} en este proyecto. 


\section{Referencias bibliográficas:}

\section{Principales ediciones y traducciones de Momus}

Boenke 1993: M. Boenke (ed., trad.), Leon Battista Alberti. Momus oder von Fürsten. Momus seu de principe (Múnich, 1993).

Brown/Knight 2003: V. Brown (ed.) y S. Knight (ed., trad.), Leon Battista Alberti. Momus (Cambridge, MA, 2003).

Consolo 1986: R. Consolo (ed., trad.), Leon Battista Alberti. Momo o del principe (Génova, 1986).

Flores Militello 2017: V. Flores Militello (trad.) y A. Mangel (ensayo), Leon Battista Alberti. Momo (Ciudad de México, 2017).

Furlan et al. 2007: F. Furlan (ed.), P. d'Alessandro (ed.) y M. Martelli (trad.), Leon Battista Alberti. Momo (Milán, 2007).

Laurens 2017: C. Laurens (trad.), Leon Battista Alberti. Momus ou le Prince. Fable politique (Paris, 2017²).

Martini 1942: G. Martini (ed., trad.), Leon Battista Alberti. Momo o del principe (Bolonia, 1942).

Medina Reinón 2002: P. Medina Reinón (trad.), L.B. Alberti. Momo o del príncipe (Murcia, 2002).

\section{Bibliografía secundaria}

Baumbach 2002: M. Baumbach, Lukian in Deutschland. Eine Forschungs- und Rezeptionsgeschichtliche Analyse vom Humanismus bis zur Gegenwart (Múnich, 2002).

Baumbach/von Möllendorf 2017: M. Baumbach y P. von Möllendorf, Ein literarischer Prometheus. Lukian aus Samosata und die Zweite Sophistik (Heidelberg, 2017).

Berti 1985: E. Berti, "Uno scriba greco-latino: il codice Vat. Urb. Lat.gr. 121 e la prima versione del Caronte di Luciano", Rivista di filologia e di istruzione classica 113 (1985), pp. 416-443.

Berti 1987: E. Berti ,“Alla scuola di Manuele Crisolora. Lettura e commento di Luciano", Rinascimento 27 (1987), pp. 3-73.

Berti 2006: E. Berti, Luciano di Samosata. Caronte. Timone. Le prime traduzioni (Florencia, 2006).

Braun 1994: E. Braun, Lukian. Unter doppelter Anklage. Ein Kommentar (Fráncfort, 1994).

Brusuelas 2009: J. Brusuelas, Recensión de Hopkins 2008, Bryn Mawr Classical Review 2009.08.11 (http://bmcr.brynmawr.edu/2009/2009-08-11.html)

Camerotto 2014: A. Camerotto, Gli occhi e la lingua della satira. Studi sull'eroe satirico in Luciano di Samosata (Milán, Udine, 2014). 
De Faveri 2002: L. De Faveri, Le traduzioni di Luciano in Italia nel XV e XVI secolo, (Ámsterdam, 2002).

Deriu 2017: M. Deriu, Mixis e poikilia nei protagonisti della satira. Studi sugli archetipi comico e platonico nei dialoghi di Luciano di Samosata (Trento, 2017).

Deriu 2018: M. Deriu, Recensión de Marquis/Billault 2017, The Classical Review 68.2 (2018), pp. 381-384.

Ferrari 2013: M. Ferrari, „Lateinische Literatur des Spätmittelalters und der Frühen Neuzeit“" [en H. R. Brittnacher y M. Ray (eds.), Phantastik: Ein interdisziplinäres Handbuch (Stuttgart, Weimar, 2013)], pp. 27-33.

Grafton 2000: A. Grafton, Leon Battista Alberti. Master Builder of the Italian Renaissance (Nueva York, 2000).

Hafner 2016: M. Hafner, Recensión de CAMERotTo 2014, Anzeiger für die Altertumswissenschaft 69 (Enero-Abril 2016), pp. 23-27.

Hafner 2017a: M. Hafner, Lukians Schrift „,Das traurige Los der Gelehrten “. Einführung und Kommentar $z u$ De Mercede Conductis Potentium Familiaribus, lib. 36 (Stuttgart, 2017).

Hafner 2017b: M. Hafner, Lukians Apologie (Tubinga 2017).

Hopkins 2008: N. Hopkins, Lucian. A Selection (Cambridge, 2008).

Iglesias Zoido 2011: J. C. Iglesias Zoido, "El nuevo Luciano de Eugenio Espejo (1747-1795): Nuevas apreciaciones sobre su legado clásico”, Dieciocho 34 (2011), pp. 225-246.

Ligota/Panizza 2007: C. Ligota y L. Panizza (eds.), Lucian of Samosata Vivus et Redivivus (Londres, Turín, 2007).

Marquis/Billault 2017: É. Marquis y A. Billault, Mixis. Le mélange des genres chez Lucien de Samosate (Paris, 2017).

Marsh 1980: D. Marsh, The Quattrocento Dialogue. Classical Tradition and Humanist Innovation, (Cambridge, MA, Londres, 1980).

Marsh 1998: D. Marsh, Lucian and the Latins. Humour and Humanism in the Early Renaissance (Ann Harbor, MI, 1998).

Marsh 2002: D. Marsh, "Review Essay: Leon Battista Alberti at the Millennium", Renaissance Quarterly 55 (2002), pp. 1028-1037.

Marsh 2004: D. Marsh, Renaissance fables: Aesopic prose by Leon Battista Alberti, Bartolomeo Scala, Leonardo Da Vinci, Bernadino Baldi (Tempe, AZ, 2004).

Marsh 2009: D. Marsh, Recensión de Ligota/Panizza 2007, International Journal of Classical Tradition 16 (2009), pp. 124-128.

Mattioli 1980: E. Mattioli, Luciano e l’Umanesimo (Nápoles, 1980).

Ní Mheallaigh 2014: K. N. Ní Mheallaigh, Reading Fiction with Lucian: Fakes Freaks and Hyperreality (Cambridge, 2014). 
Reeve 1992: M. Reeve, "The Rediscovery of Classical Texts in the Renaissance"

[en O. Pecere (ed.), Itinerari dei testi antichi (Roma, 1992)], pp. 115-157.

Reeve 1996: M. Reeve, "Classical scholarship" [en J. Kraye (ed.), The

Cambridge Companion to Renaissance Humanism (Cambridge, 1996)], pp. 20-46.

Rinaldi 1986-87: R. Rinaldi, "Parodia come allegoria: il 'Momus' di Alberti e la parodia classica”, Atti e memorie dell'Arcadia 8 (1986-87), pp. 129163.

Rinaldi 1999: R. Rinaldi, "«Momus Christianus»: Altre fonti albertiane", Lettere Italiane 51 (1999), pp. 197-252.

Robinson 1979: C. Robinson, Lucian and His Influence in Europe (Londres, 1979).

Rochette 1997: B. Rochette, Le latin dans le monde grec: Recherches sur la diffusion de la langue et des lettres latines dans les provinces hellénophones de l'Empire romain (Bruselas, 1997).

Stramaglia et al. 2016: A. Stramaglia, S. Grazzini y G. Dimatteo (eds.), Giovenale tra storia, poesia e ideologia (Berlín, Boston, 2016).

Vian Herrero 2015: A. Vian Herrero, "El exilio de la Virtud. Textos espurios en el corpus lucianeo de los siglos XV-XVI y su influencia literaria: Alberti, Vegio y sus derivados entre España e Italia”, eHumanista 29 (2015), pp. 168-207.

Vives Coll 1959: A. Vives Coll, Luciano de Samosata en España (Valladolid, 1959).

Wulfram 2012: H. Wulfram, "Ein Heilsbringer aus dem Osten. Manuel Chrysoloras und seine Entindividualisierung im italienischen Frühhumanismus“ [en F. Kovolou (ed.), Byzanzrezeption in Europa (Berlín, Boston, 2012)], pp. 89-116.

Zappala 1990: M. O. Zappala, Lucian of Samosata in the Two Hesperias. An Essay in Literary and Cultural Translation (Potomac, MD, 1990). 